\title{
THE STABILITY OF VISCOUS FLOW BETWEEN ROTATING CYLINDERS*
}

\author{
BY \\ H. STEINMAN \\ Yerkes Observatory, University of Chicago
}

\begin{abstract}
In this paper Chandrasekhar's method of solving the characteristic value problem in the theory of the stability of viscous flow between rotating cylinders is carried to a higher approximation. A comparison between the results of the various calculations among themselves and with the experiments confirms the greater feasibility of the present method.
\end{abstract}

I. Introduction. Recently S. Chandrasekhar (1954) [1] has investigated the problem of the stability of viscous flow between two concentric rotating cylinders. He employed a modified and more general method than had previously been used. The problem was first studied extensively by G. I. Taylor (1923). Later D. Meksyn (1946) expanded the theoretical treatment to cover a different range of values for the ratio of angular velocities. The significance of Chandrasekhar's method lies in its relative simplicity and its ability to treat the widest range of values for the relevant parameters. This paper is an extension of Chandrasekhar's work to obtain the solution of the problem correct to the second order in $y / d$, where $y$ is the distance of a point from the inner cylinder and $d$ is the difference in the radii of the cylinders.

The stationary solution of the hydrodynamical equations appropriate to the problem on hand is

$$
V(r)=A r+\frac{B}{r},
$$

where $V(r)$ is the rotational velocity at a distance $r$ from the axis of rotation; $A$ and $B$ are two constants related to the angular velocities $\Omega_{1}$ and $\Omega_{2}$ with which the cylinders of radii $R_{1}$ and $R_{2}$ are rotated by

and

$$
A=\Omega_{1} \frac{1-\mu R_{2}^{2} / R_{1}^{2}}{1-R_{2}^{2} / R_{1}^{2}} \quad \text { and } \quad B=\Omega_{1} \frac{R_{1}^{2}(1-\mu)}{1-R_{1}^{2} / R_{2}^{2}},
$$

$$
\mu=\Omega_{2} / \Omega_{1}
$$

is the ratio of the angular velocities.

By considering a symmetric perturbation of solution (1) by a periodic disturbance in the direction parallel to the axis of rotation with a wave length $\lambda$, a characteristic value problem in a differential equation of order six is obtained (see Chandrasekhar, loc. cit., Eq. (4)). When the difference in radii of the two cylinders is small compared to their mean, we may, to the first order in $y / d$, replace $A+B / r^{2}$ by

where

$$
A+B / r^{2}=\Omega_{1}[1-(1-\mu) \xi],
$$

$$
\xi=y / d=\frac{r-R_{1}}{R_{2}-R_{1}} .
$$

'Received February 3, 1955. 
This is the approximation on which both Meksyn and Chandrasekhar have solved the characteristic value problem. To the second order in $y / d$ we have instead

$$
A+B / r^{2}=\Omega_{1}\left[1-(1-\mu)\left\{1+\frac{3}{2} \frac{\left(R_{2}-R_{1}\right)}{R_{1}}\right\} \xi+\frac{3}{2}(1-\mu) \frac{\left(R_{2}-R_{1}\right)}{R_{1}} \xi^{2}\right] ;
$$

and we shall write this expression in the form

where

$$
A+B / r^{2}=\Omega_{1}\left[1+\alpha \xi+\beta \xi^{2}\right]
$$

$$
\begin{aligned}
\alpha & =(\mu-1)\left[1+\frac{3}{2} \frac{\left(R_{2}-R_{1}\right)}{R_{1}}\right], \\
\beta & =\frac{3}{2}(1-\mu) \frac{\left(R_{2}-R_{1}\right)}{R_{1}}, \\
\mu & =1+\alpha+\beta .
\end{aligned}
$$

With $A+B / r^{2}$ given by Eq. (6) the differential equation governing the problem is (see Chandrasekhar, Eq. (10))

where

$$
\left(D^{2}-a^{2}\right)^{3} v=-a^{2} T\left(1+\alpha \xi+\beta \xi^{2}\right) v
$$

and

$$
D=d / d \xi, \quad \lambda=a\left(R_{2}-R_{1}\right)
$$

$$
T=-\frac{4 A \Omega_{1} d^{4}}{\nu^{2}}
$$

is the Taylor number. (In Eq. (10) $\nu$ is the kinematic viscosity.) The six boundary conditions with respect to which Eq. (9) must be solved are

$$
v=\left(D^{2}-a^{2}\right) v=D\left(D^{2}-a^{2}\right) v=0 \quad \text { at } \quad \xi=0 \quad \text { and } \quad \xi=1 .
$$

Equation (9) together with the boundary conditions (11) determine $T$ for any assigned $a^{2}$. The critical Taylor number $T_{c}$, at which instability sets in is then the minimum value of $T$ as a function of $a^{2}$.

II. The revised method. Chandrasekhar's method of solving the basic characteristic value problem consists in representing Eq. (9) as a pair of differential equations, one of order four and the other of order two and in arranging that the boundary conditions split in the same way; the fourth order equation together with the boundary conditions which go with it is then solved exactly. Thus, with the same substitutions as Chandrasekhar (loc. cit., Eqs. (16)-(24)), we reduce the characteristic value problem to solving the pair of equations

and

$$
\left(D^{2}-a^{2}\right)^{2} W=\left(1+\alpha \xi+\beta \xi^{2}\right) \Psi
$$

$$
\left(D^{2}-a^{2}\right) \Psi=-a^{2} T W,
$$

together with the boundary conditions

$$
W=D W=0, \quad \Psi=0 \quad \text { at } \quad \xi=0 \quad \text { and } \quad \xi=1 .
$$

We next expand $\Psi$ as a sine series of the form 


$$
\Psi=\sum_{n=1}^{\infty} C_{n} \sin n \pi \xi
$$

thus satisfying the boundary conditions, $\Psi=0$ at $\xi=0$ and 1 . With this expansion for $\Psi$ Eq. (12) becomes

$$
\left(D^{2}-a^{2}\right)^{2} W=\left(1+\alpha \xi+\beta \xi^{2}\right) \sum_{m=1}^{\infty} C_{m} \sin m \pi \xi,
$$

and this equation is solved exactly to satisfy the remaining four boundary conditions on $W$.

The general solution of Eq. (16) can be written in the form

$$
\begin{aligned}
W=\sum_{m=1}^{\infty} \frac{C_{m}}{\left(m^{2} \pi^{2}+a^{2}\right)}\left\{A_{1}^{(m)} \cosh a \xi\right. & +B_{1}^{(m)} \sinh a \xi+A_{2}^{(m)} \xi \cosh a \xi \\
& +B_{2}^{(m)} \xi \sinh a \xi+\left(\eta+\alpha \xi+\beta \xi^{2}\right) \sin m \pi \xi \\
& \left.+\frac{4 \pi m}{m^{2} \pi^{2}+a^{2}}(\alpha+2 \beta \xi) \cos m \pi \xi\right\},
\end{aligned}
$$

where $A_{1}^{(m)}, B_{1}^{(m)}, A_{2}^{(m)}$ and $B_{2}^{(m)}$ are constants of integrations and

$$
\eta=\frac{4 \beta\left(a^{2}-5 m^{2} \pi^{2}\right)+\left(m^{2} \pi^{2}+a^{2}\right)^{2}}{\left(m^{2} \pi^{2}+a^{2}\right)^{2}} .
$$

The conditions that $W=D W=0$ at $\xi=0$ and $\xi=1$ yield:

$$
\begin{aligned}
A_{1}^{(m)} & =-\frac{4 m \pi \alpha}{m^{2} \pi^{2}+a^{2}} \\
a B_{1}^{(m)}+A_{2}^{(m)} & =-\left(\eta+\frac{8 \beta}{m^{2} \pi^{2}+a^{2}}\right) m \pi
\end{aligned}
$$

$A_{1}^{(m)} \cosh a+B_{1}^{(m)} \sinh a+A_{2}^{(m)} \cosh a+B_{2}^{(m)} \sinh a$

$$
=(-1)^{m+1} \frac{4 m \pi}{m^{2} \pi^{2}+a^{2}}(2 \beta+\alpha),
$$

$A_{1}^{(m)} a \sinh a+B_{1}^{(m)} a \cosh a+A_{2}^{(m)}(\cosh a+a \sinh a)$

$$
+B_{2}^{(m)}(\sinh a+a \cosh a)=(-1)^{m+1}\left(\alpha+\beta+\eta+\frac{8 \beta}{m^{2} \pi^{2}+a^{2}}\right) m \pi \text {. }
$$

The solution of these equations is:

$A_{1}^{(m)}=-\frac{4 m \pi \alpha}{m^{2} \pi^{2}+a^{2}}$

$B_{1}^{(m)}=\frac{m \pi}{\Delta}\left\{a \alpha_{m}^{\prime}+(\sinh a+a \cosh a) \beta_{m}^{\prime}-(\sinh a) \gamma_{m}^{\prime}\right\}$,

$A_{2}^{(m)}=-\frac{m \pi}{\Delta}\left\{\left(\sinh ^{2} a\right) \alpha_{m}^{\prime}+\left(a \sinh a+a^{2} \cosh a\right) \beta_{m}^{\prime}-(a \sinh a) \gamma_{m}^{\prime}\right\}$,

$B_{2}^{(m)}=\frac{m \pi}{\Delta}\left\{(\sinh a \cosh a-a) \alpha_{m}^{\prime}+\left(a^{2} \sinh a\right) \beta_{m}^{\prime}-(a \cosh a-\sinh a) \gamma_{m}^{\prime}\right\}$, 
where

$$
\begin{aligned}
& \Delta=\sinh ^{2} a-a^{2}, \\
& \alpha_{m}^{\prime}=\frac{8 \beta}{m^{2} \pi^{2}+a^{2}}+\eta, \\
& \beta_{m}^{\prime}=\frac{4 \alpha}{m^{2} \pi^{2}+a^{2}}\left[(-1)^{m+1}\left(1+\frac{2 \beta}{\alpha}\right)+\cosh a\right], \\
& \gamma_{m}^{\prime}=(-1)^{m+1}\left(\alpha+\beta+\alpha_{m}^{\prime}\right)+\frac{4 \alpha}{m^{2} \pi^{2}+a^{2}}(a \sinh a) .
\end{aligned}
$$

With $\Psi$ and $W$ given by Eqs. (15) and (17), Eq. (13) becomes

$$
\begin{aligned}
\sum_{m=1}^{\infty} C_{n}\left(n^{2} \pi^{2}+a^{2}\right) \sin & n \pi \xi=T a^{2} \sum_{m=1}^{\infty} \frac{C_{m}}{\left(m^{2} \pi^{2}+a^{2}\right)^{2}}\left\{A_{1}^{(m)} \cosh a \xi\right. \\
& +B_{1}^{(m)} \sinh a \xi+A_{2}^{(m)} \xi \cosh a \xi+B_{2}^{(m)} \xi \sinh a \xi \\
& \left.+\left(\eta+\alpha \xi+\beta \xi^{2}\right) \sin m \pi \xi+\frac{4 m \pi}{m^{2} \pi^{2}+a^{2}}(\alpha+2 \beta \xi) \cos m \pi \xi\right\} .
\end{aligned}
$$

Multiplying this last equation by $\sin m \pi \xi$ and integrating over the range $\xi=0$ to $\xi=1$, we obtain

$$
\begin{aligned}
\sum_{m=1}^{\infty}\left[\frac{n \pi}{n^{2} \pi^{2}+a^{2}}\{[1\right. & \left.+(-1)^{n+1} \cosh a\right] A_{1}^{(m)}+\left[(-1)^{n+1} \sinh a\right] B_{1}^{(m)} \\
& +(-1)^{n+1}\left[\cosh a-\frac{2 a}{n^{2} \pi^{2}+a^{2}} \sinh a\right] A_{2}^{(m)} \\
+ & {\left.\left[(-1)^{n+1} \sinh a-\frac{2 a}{n^{2} \pi^{2}+a^{2}}\left(1+(-1)^{n+1} \cosh a\right)\right] B_{2}^{(m)}\right\} } \\
& \left.+\alpha x_{n, m}+\beta y_{n, m}+\frac{\eta}{2} \delta_{n, m}-\frac{1}{2}\left(n^{2} \pi^{2}+a^{2}\right)^{3} \frac{\delta_{n, m}}{a^{2} T}\right] K_{m}=0,
\end{aligned}
$$

with

$$
K_{m}=C_{m}\left(m^{2} \pi^{2}+a^{2}\right)^{-2}
$$

and

$$
\begin{aligned}
& x_{n, m}=\frac{1}{4} \quad y_{n, m}=\frac{1}{6}-\frac{1}{4 n^{2} \pi^{2}}-\frac{2}{n^{2} \pi^{2}+a^{2}}, \quad \text { if } \quad n=m \text {; } \\
& x_{n, m}=0 \quad y_{n, m}=\frac{4 n m}{n^{2}-m^{2}}\left[\frac{1}{\pi^{2}\left(n^{2}-m^{2}\right)}-\frac{2}{m^{2} \pi^{2}+a^{2}}\right], \quad \text { if } \begin{array}{l}
n \neq m \\
n+m \text { even; }
\end{array} \\
& x_{n, m}=y_{n, m}=-\frac{4 n m}{n^{2}-m^{2}}\left[\frac{1}{\pi^{2}\left(n^{2}-m^{2}\right)}-\frac{2}{m^{2} \pi^{2}+a^{2}}\right], \quad \text { if } \begin{array}{ll}
n \neq m \\
n+m \text { odd }
\end{array}
\end{aligned}
$$

Equation (23) represents a system of linear homogeneous equations. It is the vanishing of the determinant of this system that determines $T$ for any assigned $a^{2}$.

III. Numerical results. The equation determining $T$ can be written as 


$$
\begin{aligned}
\| \frac{n \pi}{n^{2} \pi^{2}+a^{2}}\{[1+ & \left.(-1)^{n+1} \cosh a\right] A_{1}^{(m)}+\left[(-1)^{n+1} \sinh a\right] B_{1}^{(m)} \\
& +(-1)^{n+1}\left[\cosh a-\frac{2 a}{n^{2} \pi^{2}+a^{2}} \sinh a\right] A_{2}^{(m)} \\
& \left.+\left[(-1)^{n+1} \sinh a-\frac{2 a}{n^{2} \pi^{2}+a^{2}}\left(1+(-1)^{n+1} \cosh a\right)\right] B_{2}^{(m)}\right\} \\
& +\alpha x_{n, m}+\beta y_{n, m}+\frac{\eta}{2} \delta_{n, m}-\frac{1}{2}\left(n^{2} \pi^{2}+a^{2}\right)^{3} \frac{\delta_{n, m}}{a^{2} T} \|=0 .
\end{aligned}
$$

In solving the infinite determinantal equation (26), the determinant was terminated after three rows and columns. For $\alpha$ between -1.00 and -2.00 this seemed justified. For $\alpha<-2.00$, the calculations indicate that a higher approximation, by terminating

TABLE 1

Cylinder ratios and values of $\alpha$ with their corresponding $\beta$ and $\mu$.

$\begin{array}{lccccr}\text { Case } & R_{2} / R_{1} & d=R_{2}-R_{1} & \alpha & \beta & \mu \\ \text { I } & \frac{4.035}{3.00}=1.345 & 1.035 & -.75 & .2557 & .5058 \\ \text { I } & 1.345 & 1.035 & -1.0 & .3410 & .3410 \\ \text { I } & 1.345 & 1.035 & -1.5 & .5115 & .0115 \\ \text { II } & \frac{4.035}{3.80}=1.062 & .235 & -.75 & .0636 & .3136 \\ \text { II } & 1.062 & .235 & -1.0 & .0848 & .0848 \\ \text { II } & 1.062 & .235 & -1.5 & .1272 & -.3727 \\ \text { III } & \frac{4.035}{3.55}=1.137 & .485 & -.75 & .1276 & .3775 \\ \text { III } & 1.137 & .485 & -1.0 & .1700 & .1700 \\ \text { III } & 1.137 & .485 & -1.5 & .2551 & -.2449\end{array}$

the determinant after the fourth row and column, would be necessary. However, for the cases computed the convergence is fairly rapid as will be seen from Table 2.

In the present calculations, three values of $\alpha$ were chosen which correspond to the

\begin{tabular}{|c|c|c|c|c|c|c|c|}
\hline Case & $a$ & $\alpha$ & $\beta$ & $T_{1}$ & $T_{2}$ & $T_{2}$ & $T_{3} / T_{2}$ \\
\hline I & 3.12 & -.75 & .2557 & 2464.84 & 2462.38 & 2453.04 & .9962 \\
\hline II & 3.12 & -.75 & .0636 & 2868.86 & 2662.94 & 2652.28 & .8960 \\
\hline III & 3.12 & -.75 & .1276 & 2597.24 & 2592.75 & 2582.54 & .9861 \\
\hline I & 3.12 & -1.0 & .3410 & 2885.15 & 2878.25 & 2867.97 & .9964 \\
\hline II & 3.12 & -1.0 & .0848 & 3276.10 & 3256.78 & 3244.32 & .9962 \\
\hline III & 3.12 & -1.0 & .1700 & 3134.83 & 3120.86 & 3109.20 & .8963 \\
\hline$I$ & 3.20 & -1.5 & .5115 & 4383.32 & 4328.23 & 4316.76 & .9973 \\
\hline II & 3.20 & -1.5 & .1272 & 6018.53 & 5761.04 & 5747.24 & .9976 \\
\hline III & 3.20 & -1.5 & .2115 & 5353.70 & 5198.56 & 5181.98 & .9968 \\
\hline
\end{tabular}
experimental cases studied by Taylor; these cases are denoted by I, II and III in the

TABLE 2

Taylor numbers for assigned values of $a, \alpha$ and $\beta$. 
subsequent work. For these three values of $\alpha$, Eq. (8) leads to three values of $\beta$ and $\mu$. Case II gives the smallest separation of the cylinders and hence should be approximated best by the theory. Case $I$ is for the widest separation of the cylinders.

Table 1 gives the ratio for each case, the value of $d$ and the three values of $\alpha$ with their corresponding $\beta$ and $\mu$.

Table 2 lists the Taylor numbers calculated for the nine values of $\beta$. Here the subscript on $T$ indicates the order of the determinant which was made equal to zero in the computation of $T_{c}$. The final column gives the ratio of $T_{3}$ to $T_{2}$ and provides a measure of the convergence of the method.

The critical values of $a$ for these cases agree exactly with those used in the first approximation of $y / d$. Therefore it was possible to use some of Miss Elbert's computations. This was accomplished by writing the constants of integration in the forms

$$
\begin{aligned}
& A_{1}^{(m)}=A_{1 f}^{(m)}, \\
& B_{1}^{(m)}=B_{1 f}^{(m)}+\frac{m \pi \beta}{\Delta}\left\{\left(a+(-1)^{m+1} \cosh a\right) \frac{8}{m^{2} \pi^{2}+a^{2}}\right. \\
& \left.+\left(a-(-1)^{m+1} \sinh a\right) \frac{4\left(a^{2}-5 m^{2} \pi^{2}\right)}{\left(m^{2} \pi^{2}+a^{2}\right)^{2}}-(-1)^{m+1} \sinh a\right\}, \\
& A_{2}^{(m)}=A_{2 f}^{(m)}-\frac{m \pi \beta}{\Delta}\left\{\left(\sinh ^{2} a+(-1)^{m+1} a^{2} \cosh a\right) \frac{8}{m^{2} \pi^{2}+a^{2}}\right. \\
& \left.+\left(\sinh ^{2} a-(-1)^{m+1} a \sinh a\right) \frac{4\left(a^{2}-5 m^{2} \pi^{2}\right)}{\left(m^{2} \pi^{2}+a^{2}\right)^{2}}-(-1)^{m+1} a \sinh a\right\}, \\
& B_{2}^{(m)}=B_{2 f}^{(m)}+\frac{m \pi \beta}{\Delta}\left\{\left(\sinh a \cosh a-a+(-1)^{m+1} \sinh a\right.\right. \\
& \left.-(-1)^{m+1} a \cosh a\right)\left(\frac{8}{m^{2} \pi^{2}+a^{2}}+\frac{4\left(a^{2}-5 m^{2} \pi^{2}\right)}{\left(m^{2} \pi^{2}+a^{2}\right)^{2}}\right) \\
& \left.+(-1)^{m+1} a^{2} \sinh a\left(\frac{8}{m^{2} \pi^{2}+a^{2}}\right)-(-1)^{m+1}(a \cosh a-\sinh a)\right\} \text {, }
\end{aligned}
$$

where the subscript $f$ denotes the constants from the first order calculation. Obviously the corresponding value of $\mu$ varies. When $\alpha$ has the value -2.00 or smaller the critical value of $a$ no longer agrees with that found in the first approximation but rather appears to be consistently smaller.

A comparison of the values derived in this paper with those obtained by both Taylor and Chandrasekhar is made in Table 3. The experimental results are given in units of $\Omega_{1} / \nu$ with possible deviations of one or two per cent in the range considered. Equation (28) was used to convert the computed Taylor numbers to the unit of measure $\Omega_{1} / \nu$;

$$
\left(\frac{\Omega_{1}}{\nu}\right)^{2}=\frac{-T\left(1-R_{2}^{2} / R_{1}^{2}\right)}{4 d^{4}\left(1-\mu R_{2}^{2} / R_{1}^{2}\right)} .
$$

In this table the first column lists the case; the second, the value of $\mu$; and the last four columns the values of $\Omega_{1} / \nu$ from Chandrasekhar's first order calculation, from the nearest second order calculation, and from Taylor's theoretical work and lastly from 
TABLE 3

Comparison of values of $\Omega_{1} / \nu$ derived from the first and second order calculations using Chandrasekhar's method, from T'aylor's theoretical work and from Taylor's experiments.

\begin{tabular}{lrrrrr} 
Case & \multicolumn{1}{c}{$\mu$} & $\Omega_{1} / \nu_{\text {first }}$ & $\Omega_{1} / \nu_{\text {second }}$ & $\Omega_{1} / \nu_{\text {Taylor }}$ & $\Omega_{1} / \nu_{\text {experiment }}$ \\
I & .5058 & 68.6 & 71.3 & 77.4 & 74.2 \\
I & .3410 & 34.2 & 36.3 & 36.6 & 39.4 \\
I & .0115 & 27.3 & 31.0 & 27.8 & 30.5 \\
II & .3136 & 204.9 & 207.5 & 207.2 & 203.0 \\
II & .0848 & 190.6 & 194.0 & 193.9 & 193.8 \\
II & -.3727 & 197.2 & 206.0 & 203.7 & $\mathbf{2 1 2 . 2}$ \\
III & .3775 & 79.9 & 81.7 & 81.5 & 86.8 \\
III & .1700 & 70.2 & 72.6 & 72.8 & $\mathbf{7 4 . 6}$ \\
III & -.2449 & 66.8 & 72.2 & 71.6 & $\mathbf{7 3 . 1}$
\end{tabular}

Taylor's experiments. Both the Taylor values and the first order values had to be interpolated.

It is evident from Table 3 that in all cases the second order calculation gives a value that is larger and closer to the experimental value than the first order calculation. It is also true that for the particular cases considered the second order value is more often nearer to the experimental values than is Taylor's value. As $\alpha \rightarrow-\infty$ the Taylor theoretical values will deviate more and more from the experimental values. It seems that a third order calculation would add a correction of the right magnitude. However, this would probably require the inclusion of the second order terms in $\mu$.

The author is indebted to Dr. S. Chandrasekhar for suggesting this problem and for his valuable advice. Thanks are also extended to Dr. Chandrasekhar and Miss Donna Elbert for the use of their first order computations.

\section{REFERENCES}

1. S. Chandrasekhar, Mathematika 1, 5 (1954)

2. G. I. Taylor, Phil. Trans. Roy. Soc. A223, 289 (1923)

3. D. Meksyn, Proc. Roy. Soc. A187, 115, 480, 492 (1946) 